\title{
Estudio de la eficacia de misoprostol comparado con oxitocina, en la inducción del parto en la amenorrea prolongada
}

\author{
Jorge Mosquera; Julio César Mesa; Hernando Navarro*; Edgar Cobo**; César Neira***; Johnny Zúñiga****
}

\section{RESUMEN}

OBJETIVO: Comparar la eficacia y seguridad de la dosis de 50 microgramos de misoprostol, administrado en pincelación cervical, con dosis convencionales de oxitocina, vía intravenosa (iv.) continua, en pacientes con amenorrea prolongada y cervix desfavorable.

DISEÑO DEL ESTUDIO: Se hizo un estudio prospectivo aleatorizado y controlado. La variable primaria de salida fue el intervalo entre la iniciación de la inducción y la expulsión del feto. Las variables secundarias son los intervalos en los cuales se puede subdividir la variable primaria: intervalo entre iniciación de la inducción y la iniciación del parto, la realización de la amniotomia y la iniciación del período expulsivo; así como: la aparición de hemorragias, hiperactividad uterina, sufrimiento fetal, índice de Apgar del recién nacido y peso fetal al nacer.

RESULTADOS: Se estudiaron 89 pacientes, distribuidas así: 47 en el grupo que recibió misoprostol y 42 cn eỉ grupo que recibió oxitocina. No se encontraron diferencias significativas entre los dos grupos estudiados. La fase latente del parto fue dos horas más corta en el grupo de misoprostol, $(x=8.16 \pm 1.05$ h.) vs. oxitocina $(x=10.29 \pm 1.5$ h. $)$, diferencia que no fue estadísticamente significativa (p: 0.1401$)$.

CONCLUSION: Siendo la administración de misoprostol de más fácil aplicación, menor costo, y tan eficaz como la oxitocina iv. en la inducción del parto, en casos de amenorrea prolongada, resulta aconsejable su uso.

PALABRAS CLAVES: Eficacia, misoprostol, oxitocina, RCT, inducción de la labor.

\section{SUMMARY}

A randomized clinical trial was done, in order to compare the effectiviness and safety of misoprostol. Intravaginal doses of 50 micrograms $(\mathrm{mcg})$ were applied to the patients randomly allocated to experimental groups. Control patients received conventional doses of oxytocin, administered by continuous intravenous infusion. The primary output measure was the time interval between the begining of the induction procedure and the expulsion of the fetus (II - P). Secondary output measures are described in the full paper.

The 89 patiens studied were distributed as follows: 47 received misoprostol and 42 received oxytocin. Four patients allocated to the misoprostol group were excluded: one received oxytocin plus misoprostol by decision of the attending obstetrician and three abandoned the trial for personal decision. The Il-P time interval was $15.38 \pm 1.53$ hours for misoprostol and $18.56 \pm 1.95$ hours for oxytocin. Neither the differences between the two groups regarding the primary outcome measure, nor the differences in any of all the secundary output measures, were statistically significant. Accordingly, the use of misoprostol is recommended for induction of labor in cases of prolonged amenorrhea, considering that it is less expensive, easier to apply and safe.

KEY WORDS: Efficacy, misoprostol, oxitocyn, induction of labor, RCT.

\section{Introducción}

La gestación prolongada es una entidad obstétrica en la cual se eleva la morbimortalidad perinatal (1-3). Su

\footnotetext{
* Profesor hora cátedra, Depto. de Obstetricia y Ginecología, Escuela de Medicina, Universidad del Valle.

** Profesor Emérito de Obstetricia y Ginecologia, Escuela de Medicina, Universidad del Valle. Jefe del Depto. de Obstetricia y Ginecologia. Fundación Clínica Valle del Lili.

*** Instructor de Ecografía, Depto. de Obstetricia y Ginecologia, Escuela de Medicina, Universidad del Valle.

**** Ginecobstetra. Universidad del Valle.
}

manejo requiere inducción del parto, casi siempre con un cervix desfavorable. El método convencional es la administración de oxitocina, que cuando el cervix es favorable, (indice de Bishop > 9), muestra resultados exitosos.

Cuando el índice de Bishop es menor de 9, el resultado no es tan favorable y se observa una alta proporción de inducciones fallidas, que lleva a un aumento en las tasas de operación cesárea.

Algunas prostaglandinas aumentan la contractilidad uterina y al mismo tiempo aceleran la maduración cervical. El misoprostol es un análogo sintético de la prostaglandina E-1 (PGE 1), empleada como terapéutica en afecciones de la mucosa gastroduodenal (4-5) y más 
recientemente estudiada en obstetricia como estimulante de la contractilidad uterina y de la maduración del cervix, (6-13), se han encontrado pocos efectos colaterales adversos para la madre o para el feto.

Como una medida tendiente a reducir la indicación de cesárea por inducción fallida, iniciamos en 1993 un ensayo clínico controlado que nos permitiera evaluar comparativamente la eficacia del misoprostol y la oxitocina en casos de gestación prolongada y cervix desfavorable.

\section{Pacientes y métodos}

El estudio se realizó desde el 2 de abril de 1993 hasta el 2 de abril de 1996, en el Hospital Universitario del Valle (HUV), centro de referencia de tercer nivel, localizado en Cali, Colombia, que cubre una población de alto riesgo del sur occidente del país y es, además, el Hospital docente de la Escuela de Medicina de la Universidad del Valle.

Los criterios de inclusión fueron: 1. Amenorrea prolongada de 42 o más semanas, determinada por falla menstrual confiable $\mathrm{y} / \mathrm{o}$ estudio ecográfico de la primera mitad de la gestación, 2. Indice de Bishop menor o igual a 4 y 3 . Feto único, vivo, normal, en presentación cefálica.

Los criterios de exclusión fueron: 1. Contracciones uterinas regulares, 2 . Ruptura prematura de las membranas, 3. Placenta previa o Abruptio placentae, presentación podálica o distócica, cirugía uterina previa, 4 . Sufrimiento fetal, diagnosticado clínico o electrónicamente, 5. Malformaciones fetales, 6. Gestación múltiple y 7 . Feto muerto.

Se calculó el tamaño muestral fijando una disminución del $30 \%$ en la duración del trabajo de parto, cuando se administrara misoprostol, aplicando un poder de $80 \%$ y un nivel de significancia del 0.05 . El análisis de poder posthoc (27) de las diferencias observadas entre los dos grupos, indica que con 44 pacientes por grupo se puede demostrar si estas diferencias son estadísticamente significativas. En 1983 la amenorrea prolongada se presentó en el HUV en el $2 \%$ de los embarazos. Calculando que esta tasa pudiera reducirse a $1 \%$ y teniendo en cuenta que el HUV recibe anualmente 8.000 pacientes remitidas de los centros periféricos, tendríamos aproximadamente $80 \mathrm{pa}$ cientes anuales; sin embargo, por cambios en las políticas locales de salud, la referencia de pacientes disminuyó notoriamente, teniéndose que tomar la decisión de concluir el estudio con 89 pacientes al cabo de 3 años. La aleatorización se realizó aplicando un programa estadístico computarizado para 130 números. Para asignar los grupos control y experimental (casos), se usaron sobres opacos cerrados y sellados en orden numérico secuencial, que se abrieron después de realizar la aleatorización.

El grupo control se conformó con aquellas pacientes que recibirían oxitocina en infusión iv. continua, en dosis crecientes desde una hasta 10 miliunidades por minuto $(\mathrm{mU} / \mathrm{min})$, hasta lograr una actividad uterina de fase activa del parto normal. El grupo experimental se constituyó con las pacientes que recibirían misoprostol, $1 / 4$ de tableta de $200 \mathrm{mcg},(50 \mathrm{mcg})$, la cual se disolvía en 0.5 a $1.0 \mathrm{cc}$ de solución salina y se colocaba por especuloscopia, pincelando con un escobillón esta solución alrededor del orificio cervical externo. El procedimiento fue realizado por uno de los investigadores. Ninguna paciente fue cambiada de su grupo original.

Se realizó un tacto vaginal inicial, que se repitió cada dos horas antes de la amniotomia y después a criterio de los médicos encargados de la paciente. Los datos de dilatación cervical y estación de la presentación se registraron en un partograma.

La actividad miometrial y la frecuencia cardíaca fetal fueron controladas clínica y/o electrónicamente. Las pacientes permanecieron en la sala de trabajo de parto durante 12 horas, y si no se había iniciado éste, o no se había realizado una amniotomia, fueron trasladadas a la sala de observación por 12 horas más, al cabo de las cuales si no habían iniciado trabajo de parto se repetía la aplicación de misoprostol, en el grupo experimental y se reiniciaba una nueva inducción con oxitocina en el grupo control. Si la inducción resultaba fallida en las pacientes del grupo experimental, se les administraba oxitocina iv, en infusión continua, pero sin ser incluidas en el grupo control. Las decisiones obstétricas no siempre eran tomadas por el mismo grupo que iniciaba la intervención.

Si se presentaban polisistolia y/o hipertonia uterina, se suspendía la infusión de oxitocina o se efectuaba limpieza del cervix con solución salina, según fuera el grupo asignado.

Los casos de sufrimiento fetal agudo se trataron con máscara de oxígeno y decúbito lateral izquierdo. Cuando no desaparecieron los signos de sufrimiento fetal se realizó una operación cesárea.

Los resultados se expresan en valores promedio y porcentajes. Los promedios se acompañan del error estándar de la media. Las diferencias entre los dos grupos, cuando las hubo, fueron analizadas con el programa Epi-Info 6, que permite utilizar las pruebas de Chi-cuadrado y exacta de Fisher con factor de corrección de Yates. Se consideró estadísticamente significativa una diferencia cuyo valor de $\mathrm{p}$ fuera menor de 0.05 .

Este trabajo fue aprobado por el comité de investigaciones de la Escuela de Medicina.

Las pacientes que cumplieron los criterios de inclusión y aceptaron participar, firmaron un consentimiento informado.

\section{Resultados}

Un total de 93 pacientes cumplieron los criterios de inclusión. 51 resultaron asignadas al grupo experimental y 42 al grupo control. Cuatro pacientes del grupo asignado al misoprostol fueron excluidas posteriormente: una por haber recibido misoprostol y oxitocina, por decisión del médico tratante y tres por abandono voluntario de las pacientes. Esto redujo el grupo experimental a 47 pacientes. La distribución de las pacientes por edad, gravidez, paridad y edad gestacional fueron similares en ambos grupos, como también lo fue el índice de Bishop (Tabla 1).

El intervalo entre la iniciación de la inducción y la iniciación del parto (II-IP) fue de $8.16 \pm 1.05$ horas en el grupo que recibió misoprostol y de $10.29 \pm 1.5$ horas en el grupo que recibió oxitocina. A pesar de existir una 
diferencia de 2 horas a favor del misoprostol, esta no fue estadisticamente significativa $(\mathrm{p}=0.140)$.

No hubo diferencias estadísticamente significativas en los intervalos desde el inicio de la inducción hasta el parto, como lo muestra la tabla 2.

El parto se logró en las primeras 12 horas en el $78.6 \%$ de los casos para oxitocina (33/42) y en $72.3 \%$ de los casos para misoprostol (34/47). ( $p=0.4963)$.

La tasa de cesárea en el grupo de oxitocina fue de $19 \%$ $y$ en el de misoprostol de $23.4 \%$, con $p=0.616$. La inducción fue fallida en un $2.4 \%$ de los casos para oxitocina, y en un $12.8 \%$ para misoprostol, pero a pesar de estas diferencias porcentuales, no alcanzaron significancia estadística. (Tabla 3 ).

Con relación al resultado neonatal, evaluado por la presencia de sufrimiento fetal, meconio en el líquido amniótico, el puntaje de APGAR, el peso al nacimiento menor de $2.500 \mathrm{gr}$ o mayor de 4.000 gramos, no hubo diferencias estadísticamente significativas (Tabla 3).

El partograma que inscribe la evolución de la dilatación cervical en función del tiempo, muestra una evolución del parto más corta en el grupo que recibió misoprostol,

Tabla 1

CARACTERISTICAS DE LOS GRUPOS

\begin{tabular}{|c|c|c|c|}
\hline & Grupo oxitocina & Grupo misoprostol & $\begin{array}{c}\text { Valor de } \\
\text { p (significancia) }\end{array}$ \\
\hline Edad materna & $26.8 \pm 0.7$ & $26.6 \pm 0.8$ & $0.4032 \mathrm{NS}$ \\
\hline Gravidez & $1.69 \pm 0.11$ & $1.87 \pm 1.57$ & $0.6019 \mathrm{NS}$ \\
\hline Paridad & $0.61 \pm 0.15$ & $0.45 \pm 0.46$ & $0.3480 \mathrm{NS}$ \\
\hline Indice de Bishop & $2.87 \pm 0.17$ & $2.88 \pm 0.17$ & $0.5234 \mathrm{NS}$ \\
\hline Edad gestacional & $42.1 \pm 0.9$ & $42.1 \pm 0.08$ & $0.4104 \mathrm{NS}$ \\
\hline Ballard & $40.6 \pm 0.18$ & $40.4 \pm 0.18$ & $0.4044 \mathrm{NS}$ \\
\hline
\end{tabular}

Tabla 2

INTERVALOS ESTUDIADOS DURANTE EL TRABAJO DE PARTO

\begin{tabular}{|lccr|}
\hline & Grupo oxitocina & Grupo misoprostol & $\begin{array}{c}\text { Valor de } \\
\text { p (significancia) }\end{array}$ \\
\hline II-IP & $10.29 \pm 1.5$ & $8.16 \pm 1.05$ & $0.1401 \mathrm{NS}$ \\
II-P & $7.56 \pm 1.05$ & $7.2 \pm 0.84$ & $0.3686 \mathrm{NS}$ \\
II-EX & $17.38 \pm 1.87$ & $14.48 \pm 1.56$ & $0.1739 \mathrm{NS}$ \\
II-A & $10.37 \pm 1.07$ & $11.96 \pm 1.52$ & $0.2436 \mathrm{NS}$ \\
II-P & $18.52 \pm 1.07$ & $15.38 \pm 1.53$ & $0.1047 \mathrm{NS}$ \\
\hline
\end{tabular}

II: Inicio de la inducción

IP: Inicio del trabajo de parto

EX: Inicio del 'período expulsivo

A: Amniotomia

P: Parto.

Tabla 3

RESULTADOS MATERNOPERINATALES

\begin{tabular}{|lccc|}
\hline & Grupo oxitocina & Grupo misoprostol & $\begin{array}{c}\text { Valor de } \\
\text { (significancia) }\end{array}$ \\
\hline $\begin{array}{l}\text { Puntaje de APGAR }<7 \\
\text { Peso al nacer }<\end{array}$ & $4.8 \%(2 / 42)$ & $6.7 \%(3 / 45)$ & $0.9364 \mathrm{NS}$ \\
2.500 gr & $2.4 \%(1 / 42)$ & $0 / 45$ & \\
Peso al nacer $>$ & $21.4 \%(1 / 42)$ & $6.7 \%(3 / 46)$ & \\
4.000 gr & $21.4 \%(9 / 42)$ & $21.4 \%(1 / 42)$ & $0.6912 \mathrm{NS}$ \\
Meconio & $16.7 \%(7 / 42)$ & $10.6 \%(5 / 47)$ & $0.8235 \mathrm{NS}$ \\
Sufrimiento fetal & $14.2 \%(6 / 42)$ & $10.6 \%(5 / 47)$ & $0.4058 \mathrm{NS}$ \\
Hiperactividad uterina & $78.6 \%(33 / 42)$ & $72.3 \%(34 / 47)$ & $0.6017 \mathrm{NS}$ \\
Parto vaginal £ 12 hr & $19.0 \%(8 / 42)$ & $23.4 \%(11 / 47)$ & $0.4963 \mathrm{NS}$ \\
Cesárea & $2.4 \%(1 / 42)$ & $11.8 \%(6 / 47)$ & $0.6166 \mathrm{NS}$ \\
Inducción fallida & & & $0.1548 \mathrm{NS}$ \\
\hline
\end{tabular}


aunque la velocidad de la dilatación cervical es sensiblemente igual a la de la oxitocina (Figura 1). Al excluir la fase latente de los dos partogramas (Figura 2), las curvas se aprecian prácticamente superpuestas, mostrando que las diferenciaba; era la duración de la fase latente del parto.

La forma de estas curvas corresponde a una exponencial simple y creciente; en nuestro medio el partograma se hace siguiendo la curva sigmoidea descrita por Friedman (26), que incluye una fase final de desaceleración de la velocidad de dilatación cervical.

Nosotros hubiéramos obtenido curvas sigmoideas si se hubieran realizado tactos vaginales más frecuentes. Dicho de otra forma estas curvas exponenciales simples son un segmento de las sigmoideas.

\section{Discusión}

El uso de las prostaglandinas como método de inducción del parto, se encuentra ampliamente estudiado, particularmente la prostaglandina E2, que se encuentra disponible en el país, en forma de tabletas para administración por vía oral $\mathrm{y}$ también en tabletas vaginales $(12,14-15,22)$.
Los estudios aleatorizados que comparan la PGE2 con la oxitocina en infusión endovenosa continua, demuestran en términos generales igual eficacia, existiendo algunas diferencias en la vía de administración y presentación de efectos adversos (13-14).

El misoprostol, análogo sintético de la PG E-1, comercializado para el tratamiento de la enfermedad ulceropéptica (4-5), se ha estado utilizando en protocolos experimentales en ginecobstetricia, basándose en la hipótesis que su semejanza estructural con las prostaglandinas debe reflejarse en efectos clínicos similares. Existen diversos informes del uso de misoprostol en la inducción del aborto en el embarazo de primer y segundo trimestre (6-8); otros estudios comparativos de misoprostol vs oxitocina (16-21) y misoprostol vs dinoprostona (11-12), usados en general localmente y por la vía oral para la inducción en caso de la ruptura prematura de las membranas ovulares (13). Casi todos estos trabajos se caracterizan por estudiar muestras pequeñas y generalmente incluyen en el mismo grupo, diferentes indicaciones para la inducción del trabajo de parto.

Nuestros resultados deben ser analizados considerando que en la literatura solo hay un trabajo que estudia

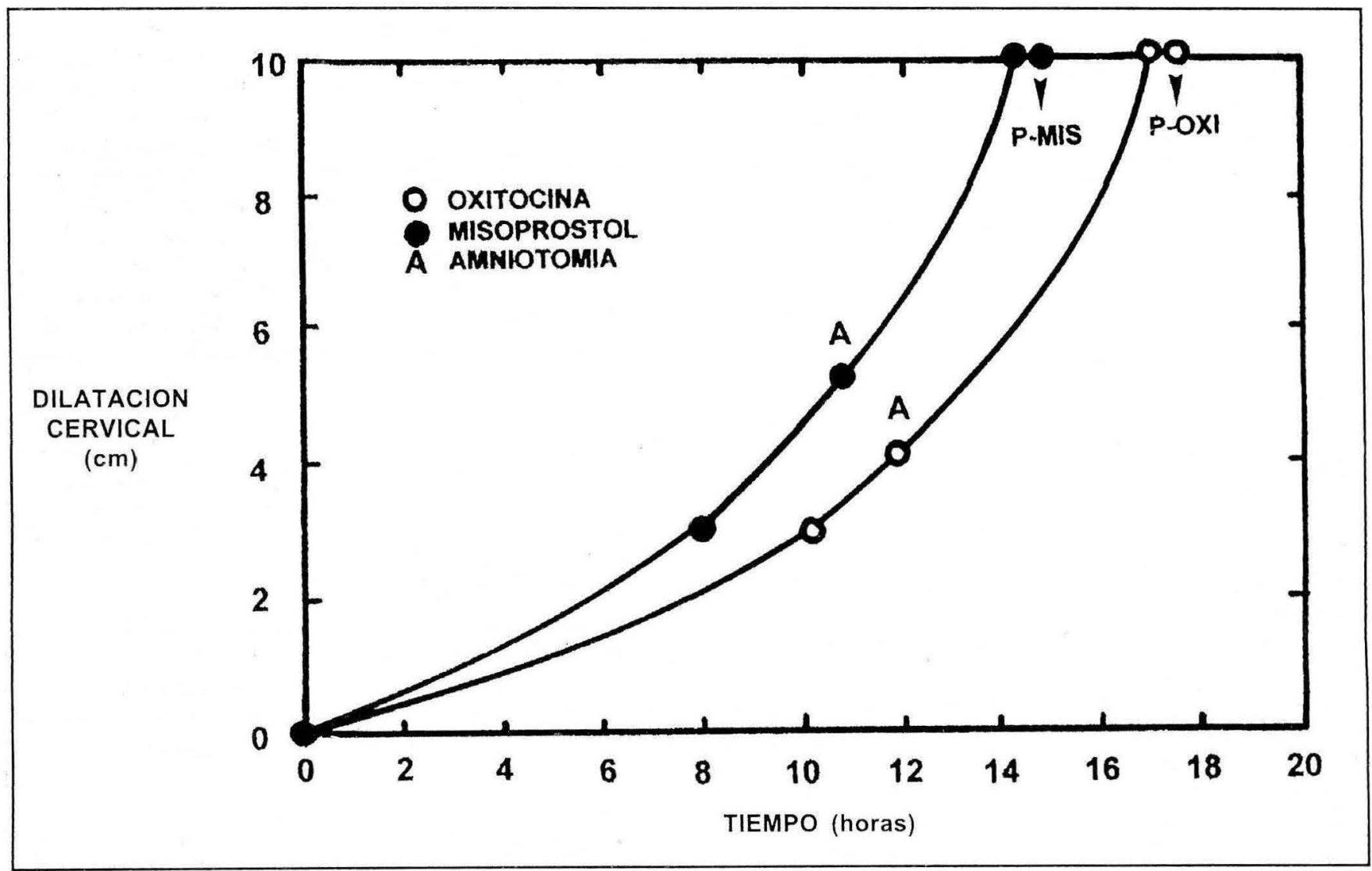

\section{Figura 0001 i01 \\ CURVAS PROMEDIO DE DILATACION CERVICAL EN FUNCION DEL TIEMPO EN LOS DOS GRUPOS ESTUDIADOS}

La curva de la izquierda muestra la evolución de la dilatación cervical desde el comienzo de la inducción, hasta el final del período expulsivo, en los casos que recibicron misoprostol, y la de la derecha los controles a quienes se administró oxitocina. Se observa como el parto ocurre casi tres horas antes con misoprostol (P-MIS), que con oxitocina (P-OXI). 


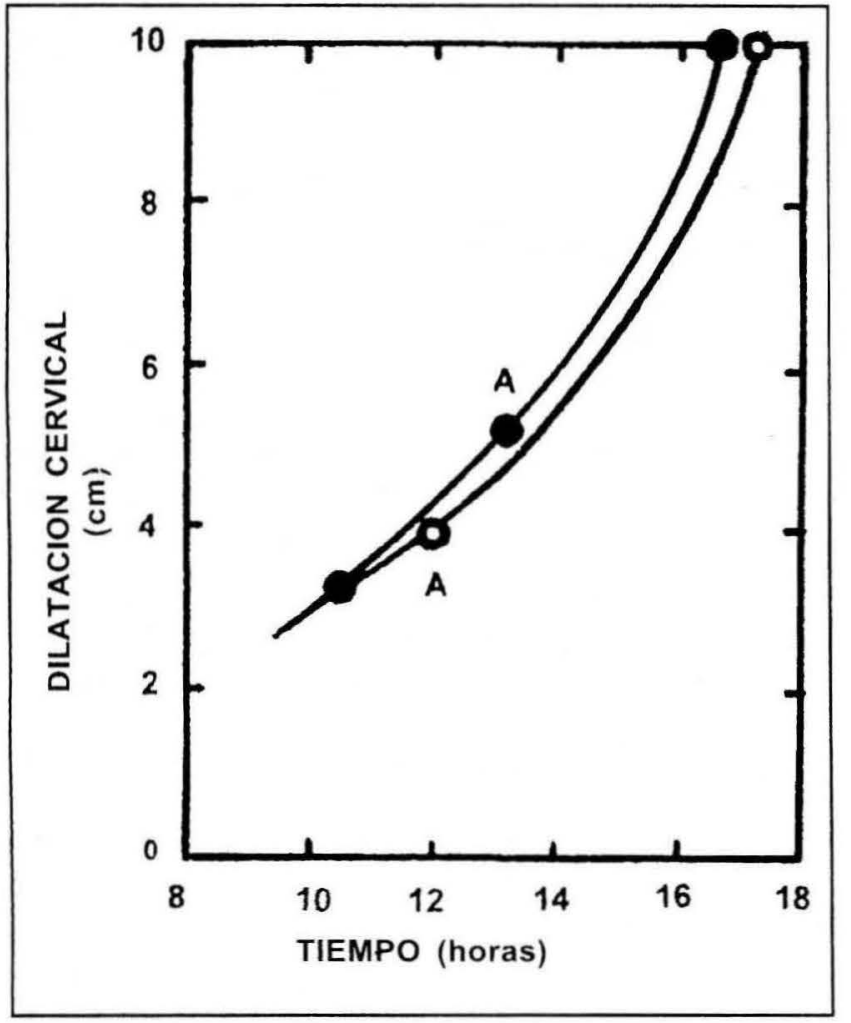

Figura 0001 i02

La misma forma de representación de la figura 1. Aqui se ha excluido el período de dilatación de 0 a $3 \mathrm{~cm}$ (fase latente del parto), observándose como a partir de la iniciación del trabajo de parto inducido (fase activa), las dos curvas son sensiblemente iguales.

misoprostol en casos de amenorrea prolongada (19), aunque anotamos que nuestro estudio comenzó un año antes (1993), pero que la captación de pacientes resulto más lenta de lo programada y por ello esta publicación aparece después. Nuestros hallazgos coinciden con la mayoría de los autores que han publicado sus resultados en los últimos dos años, en cuanto a que no hay diferencias significativas en la duración del parto con misoprostol y oxitocina, aunque la tendencia es más favorable al misoprostol. $(16,18,21,25)$.

Queremos destacar que la duración de la inducción médica del parto, medida desde su iniciación, hasta la expulsión del feto, fue más de 3 horas menor en el grupo que recibió misoprostol. Este acortamiento se realizó a expensas del período latente, que fue de 2.5 horas, a diferencia de lo observado por otros autores (19), que encuentran que el intervalo entre la iniciación de la droga a la iniciación del parto fue más corto con oxitocina. Este resultado destaca el efecto del misoprostol sobre la maduración del cervix desfavorable, propio del embara- zo prolongado y aceptado como el resultado de la actividad directa de las prostaglandinas, sobre las propiedades estructurales del cervix $(12,14-15)$.

Diferentes publicaciones muestran la hiperestimulación uterina como efecto colateral y adverso (polisistolia e hipertonia) $(10-12,20,23)$; en nuestro estudio no encontramos aumento de la hiperestimulación uterina, ni broncoaspiración de meconio (12); hecho que explicamos por la dosis utilizada de $50 \mathrm{mcg}$ de misoprostol cada 24 horas, a diferencia de otros estudios que administran dosis de 100 mcg o más (20). A pesar de que la dosis óptima de misoprostol no ha sido aún determinada, es probable que la variabilidad individual de respuesta sobre el cervix, permita utilizar dosis bajas, para lograr el efecto buscado sobre la dinámica cervical y miometrial, sin llegar a la hiperestimulación. Por esta razón pensamos que desde el punto de vista práctico y considerando que la amenorrea prolongada se asocia con mayor morbilidad perinatal, es aconsejable iniciar la inducción del parto con dosis bajas de misoprostol, como la usada por nosotros experimentalmente, y aumentarla hasta 100 ó 200 mcg, dependiendo de la respuesta obtenida, tal como se hace con la infusión de oxitocina, que en muchos protocolos se inicia con dosis tan bajas como $0.5 \mathrm{mU} / \mathrm{min}$ (24).

Nuestro estudio muestra un porcentaje alto de inducciones fallidas con misoprostol, $12.8 \%$, comparada con solo $2,4 \%$ con oxitocina, diferencia que aunque no resultó estadísticamente significativa, puede tener una obvia significación clínica. Creemos que este resultado puede explicarse por la dosis de $50 \mathrm{mcg}$, baja de acuerdo a los trabajos publicados en la actualidad, pero prudente para la época en que iniciamos el presente ensayo clínico, cuando no había referencias de la literatura.

Las diferencias observadas entre los dos grupos estudiados, no fueron estadísticamente significativas, y como se observa en las tablas II y III, algunas de las variables estudiadas muestran tendencias favorables a la aplicación de misoprostol y otras a la administración de oxitocina. Por lo tanto, es necesario esperar que la certeza sobre la significación estadística de cada una de estas variables, aparezca cuando se estudien grupos con muestras mayores, o estudios multicéntricos, con la misma o mejor metodología en la aplicación del ensayo clínico controlado. Peró entre tanto, puesto que la administración de misoprostol es más fácil, de menor costo y por lo menos tan eficaz como la de la oxitocina, recomendamos el uso del misoprostol, como la droga de primera elección en la inducción médica del parto en los casos de amenorrea prolongada.

\section{Agradecimientos}

Especiales agradecimientos al personal de salud del Hospital Universitario del Valle Evaristo García, al Departamento dè Obstetricia y Ginecología de la Universidad del Valle, al Profesor Edgard Cobo por su continua orientación y a PLACIRH, Programa Latinoamericano de Capacitación e Investigación en Reproducción Humana, por su apoyo financiero. 


\section{BIBLIOGRAFIA}

1. Arias F. Predictability of complications associated with prolonged pregnancy. Obstet Ginecol 1987; 70: 101-105.

2. Clifford HS. Postmaturity with placental dysfunction. Clinical syndrome and pathologic findings. J Pediat 1954; 1: 44-49.

3. Harris BA., Huddeston JF., Sutiiff G/, Pedis HW.The unfavorable cervix in prolonged pregnancy. Obstet Gynecol. 1983; 62: 171-173.

4. Garris RE., Kirwood CF. Misoprostol: A prostaglandin E-1 analogue. Clin Pharmacokinet 1989; 98: 627- 644.

5. Soutag SJ., Mazure PA., Poutes JF et al. Misoprostol in the treatment of duodenal peptic ulcer. A multicenter double-blind placebocontrolled study. Dig Dis Sci 1985; 30: 159s -163s.

6. Bugalho A., Bique C., Almeida L., Bergstrom S. Aplication of vaginal misoprostol before cervical dilatation to facilite first-trimester pregnancy interruption. Obstet Gynecol 1994; 83: 729-731.

7. Norman JE., Thong KJ., Baird DT. Uterine contraction and induction of abortion in early pregnancy by misoprostol and mifepristone. Lancet 1991; 338: 1233-1236.

8. Schaff EA., Worthman M., Eisinger S., Franks P. Methotrexate and misoprostol when surgical abortion fail. Obstetric Gynecol 1996-87450-452.

9. Sánchez L., Kaunitz A., DelValle G., Delke 1., Schroder P., Briones D. Labor induction with the prostaglandin E - 1 Methyl analogue misoprostol versus oxytocin: Arandomized trial. Obstet Ginecol 1993; 81: 332-336.

10. Campos GA., Margulies M., Voto LS., Rodriguez A., Guzman S. Misoprostol -un análogo de Pg E-1 para la inducción de parto a término-. Estudio comparativo y ramdomizado con oxitocina. Obst Ginec Lat Americ 1994; 52: 158-165.

11. Buser D., Mora G., Arias F. A Randomized comparition between misoprostol an dinoprostone for cervical ripening and labor induction in patientswith unfavorable cervices. Obstet Gynecol 1987; 89: 581-585.

12. Wing D., Jones M., Rebail A., Godwin M., Paul R. A comparison of misoprostol and prostaglandin E-2 gel for preinduction cervical ripening and labor induction. Am J Obstet Gynecol 1995; 1: 804-810.

13. Wingal S., Kee W., Lao T., Chung ho P. Cervical priming oral misoprostol in prelabor rupture of membranas at term. Obstet Gynecol 1996; 87: 927-936.
14. Norman M., Ekman G., Malmstrom A. Prostaglandin E2-induced opening of the human cervix involves changes in proteoglycan metabolism. Obstet Gynecol 1993; 82: 1013-1020.

15. Gottschal D., Borgida A., Mijalek J., Sauer F. A randomized clinical trial comparing misoprostol with prostaglandin $\mathrm{E} 2$ gel for preinduction cervical ripening. Am J Obstet Gynecol 1997; 1177: 1067-1070.

16. Kramer RL. A randomized trial of misoprostol and oxytocin for induction of labor: safety and efficacy . Obstet Gynecol 1977; 89: 387-391.

17. Feingold M. A randomized trial of misoprostol an oxytocin for induction of labor: safety and eficac (Letter). Obstet Gynecol. 1997 Jul.

18. Kadanalí S., Kucukoskan T., Zor $\mathrm{N}$ et al. Comparison of labor induction with misoprostol vs. oxytocin prostaglandin E-2 in term pregnancy. Int J Gynaecol Obst 1996; 55(2): 99-104.

19. Bugalho A., Bique C., Machungo F., Faundes A. Vaginal misoprostol as an alternative to oxytocin for induction of labor in women with late fetal death. Am J Obstet Gynecol 1994; 171: 538-541.

20. Echavarría E., Rocha M. Estudio comparativo randomizado de inducción de parto con oxitocina y misoprostol en embarazos en vias de prolongación. Rev Chil Obstet Ginecol 1995; 60: 108-111.

21. Escudero F., Contreras H. A comparative trial of induction with misoprostol versus oxytocin. Int J Gynaecol Obstet 1997; 57: 139-143.

22. Kierse MJNC. Prostaglandins in preinduction cervical ripening: metaanalysis of worldwide clinical experience. J Reprod Med 1993; 38 : 89-100.

23. Farah L., Ramos L., Rosa C., Delvalle G., Gaudier F., Deike L., Kaunitz. A. Randomized trial of two doses of the prostaglandin E-1 analogue misoprostol for labor induction. Am J Obstet Gynecol 1997; 177: 364-371.

24. American College of Obstetrics and Gynecology. Induction of labor. Washington: 1995. AGOG Technical Bulletin No. 217.

25. Windrim R., Benett K., Mundie $W$ et al. Oral administration of misoprostol for labor induction: a randomized controlled trial. Obstet Gynecol 1997; 89: 392-397.

26. Friedman EA, Sachtienben MR. Dysfuntional labor Prolonged latent phase in the nullipara. Obstet Gynecol 1961; 17: 135-140.

27. Altman DG. Practical stadistics for medical research. Chapman B.hall, London 1992; 455-458. 